\title{
When the Disorder is Just Right
}

\author{
A new model suggests that disorder can be a crucial ingredient for \\ producing non-Fermi-liquid behavior in a system of interacting fermions.
}

By Philip W. Phillips

the n 1956, physicist Lev Landau developed a theory describing the low-temperature properties of helium-3 as those of a liquid of interacting fermions. Landau's Fermi-liquid theory turned out to be widely applicable. It successfully describes, for instance, the low-temperature properties of most metals. There are, however, numerous fermionic systems that aren't Fermi liquids, including one-dimensional "Luttinger" liquids, Mott insulators, and heavy fermion materials. Understanding these exotic systems is an important research direction in modern condensed-matter physics, but their theoretical description is

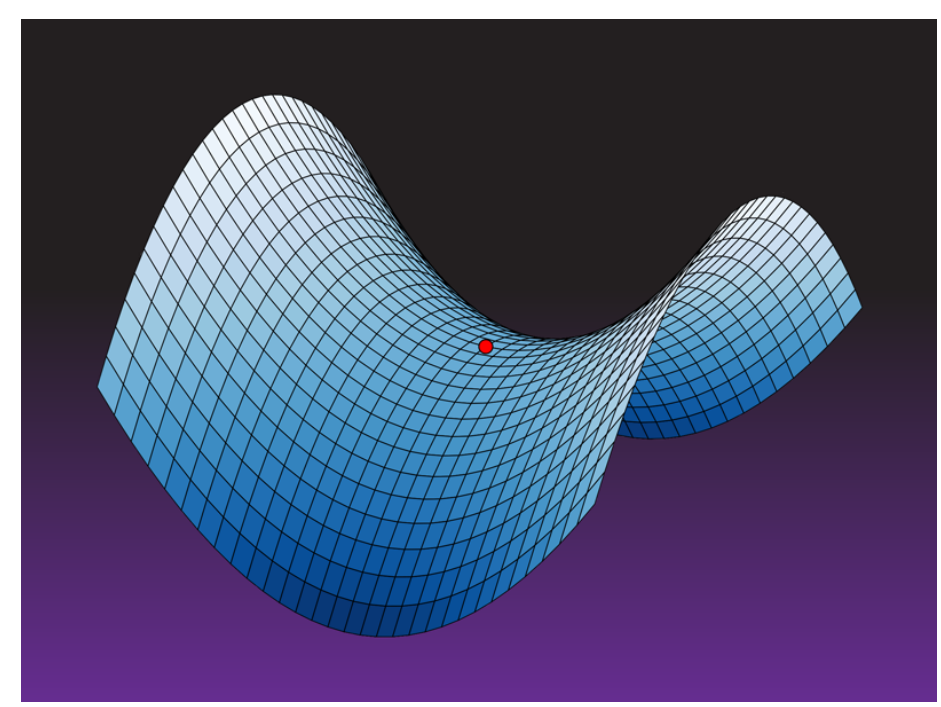

devilishly difficult. Only a handful of solvable non-Fermi-liquid models exist for simplified cases. Building on one of these models, called the Sachdev-Ye-Kitaev (SYK) model [1], a team led by Subir Sachdev of Harvard University has developed a new theory that addresses an important, unresolved question: how does non-Fermi-liquid behavior arise in a condensed-matter system-for instance, one where fermions (such as electrons) are coupled to massless bosons (such as phonons)? The team's results show that in a 2D system, non-Fermi liquid behavior can emerge if there is a sufficient degree of disorder in the coupling between the bosons and the fermions [2].

To understand what might destroy Fermi-liquid behavior, it is helpful to recall an important property of Fermi liquids-they admit a purely local description in momentum space. That is, their excitations are "quasiparticles" with well-defined momenta. As a consequence of the uncertainty principle, this localization in momentum space corresponds to delocalization in position-Fermi liquids exhibit long-range entanglement in real space. This delocalization makes Fermi liquids robust against local perturbations such as short-range repulsive Coulomb interactions. Changing the sign of the interactions, however, can break down Fermi-liquid behavior. In conventional superconductors, for instance, the coupling of electrons to phonons produces an attractive interaction-the Cooper-pairing interaction. This sign change produces the

Figure 1: Artistic rendition of a saddle point. Sachdev and co-workers have developed a new model that describes a system of fermions (such as electrons) with a random coupling to bosons (such as phonons). With a sufficient degree of disorder in the fermion-boson coupling, the model predicts the emergence of a saddle point in the fermionic bands that is associated with non-Fermi-liquid behavior.

Credit: Nicoguaro; adapted by APS/Alan Stonebraker superconducting state, which can be viewed as an instability of a Fermi liquid.

In the absence of attractive interaction, a possible strategy to find non-Fermi liquids involves introducing some sort of long-range or nonlocal interaction. Indeed, an early proposal took advantage of the long-range correlations emerging at a critical point of a quantum phase transition [3]. However, such an idea resulted in a theory that isn't "well 
behaved"[4] - violating a prescription for the construction of a low-energy theory formulated by physicist Kenneth Wilson [5].

In the early 1990s, researchers made progress by developing two exactly solvable models that use the nonlocal-interaction route to generate non-Fermi-liquid behavior. Both models invoke all-to-all interactions-each particle interacts with all other particles. The Hatsugai-Kohmoto (HK) model involves electrons hopping with a given kinetic energy between neighboring sites of a 2D lattice and includes a constant two-body interaction among all electrons that satisfy a center-of-mass constraint [6]. The result is an exactly solvable model that produces a Mott insulating phase when the interaction exceeds the width of the electronic bands $[6,7]$. To date, this approach is the only model for Mott insulators that is solvable regardless of the lattice dimensionality. The second approach is the SYK model, which contains two-body, all-to-all interaction terms that, unlike those in the HK model, are not constant but are randomly distributed. This model is exactly solvable when the number of "flavors" of fermions (fermions belonging to different bands can be regarded as having different flavors) is large. The SYK model yields clear deviations from Fermi-liquid behavior, such as an anomalous power-law scaling of the decay of the temporal correlations between particles. The original SYK model is zero dimensional (it includes time but no spatial coordinates), but extensions to higher dimensions have been proposed [8].

Both models show that nonlocal interactions, be they constant (HK) or random (SYK), are sufficient to break the long-range real-space entanglement that undergirds the quasiparticle picture of a Fermi liquid. Researchers have attempted to extend the SYK model to the case of a Fermi liquid coupled to a massless boson, but these attempts had the side effect of producing an unphysical nonzero entropy at zero temperature [9].

The theory developed by Sachdev's team avoids the entropy issue. The trick behind the researchers' result lies in a special coupling of the bosons to the fermions. In prior work, this coupling was assumed to be constant. Here, the innovation is that the coupling strength is a random variable that is tied to the boson and fermion flavors and is described by a Gaussian distribution. The best way to think about flavor-space randomness is to consider a model in which fermions in multiple bands are coupled to phonons through a band-dependent phonon coupling that is randomly distributed. Such randomness can be thought of as disorder in the system [10]. The first step in the researchers' analysis is to average over the random distribution. Assuming a large number of flavors, the team obtains a non-Fermi-liquid theory that has strong coupling between the bosons and fermions but also captures the all-to-all nature of the disorder through certain correlation functions. The researchers also find that the resulting theory has a spacetime gauge-like symmetry that leads to a vanishing of the soft mode at the critical Fermi surface (the Fermi surface at the quantum critical point). It is precisely the absence of this soft mode that makes the theory well behaved [2].

The larger picture this work paints is that getting the right theory is all about introducing the right kind of disorder. SYK by itself is insufficient to obtain such a theory. The addition of a second element-bosons coupled to fermions-to SYK is also insufficient [8]. However, adding disorder in the fermion-boson coupling might do the job. As there have been experimental proposals to test SYK physics with cold-atom experiments, perhaps the mechanism described by Sachdev's team will also motivate experimental realizations. Finally, a

thought-provoking idea is to explore the analogies between the breakdown of the Fermi-liquid quasiparticle picture and the breakdown of the particle picture hypothesized by a theory known as "unparticle physics" [11]. Could one obtain a reformulation of the boson-fermion coupling that is similar to the continuous mass formulation of unparticle physics?

Philip W. Phillips: Physics Department, University of Illinois at Urbana-Champaign, Urbana, IL, USA

\section{REFERENCES}

1. S. Sachdev and J. Ye, "Gapless spin-fluid ground state in a random quantum Heisenberg magnet," Phys. Rev. Lett. 70, 3339 (1993).

2. I. Esterlis et al., "Large- $N$ theory of critical Fermi surfaces," Phys. Rev. B 103, 235129 (2021).

3. J. A. Hertz, "Quantum critical phenomena," Phys. Rev. B 14, 1165 (1976).

4. S.-S. Lee, "Low-energy effective theory of Fermi surface coupled with $U(1)$ gauge field in $2+1$ dimensions," Phys. Rev. B 80, 165102 (2009).

5. Wilson showed that obtaining a proper low-energy theory 
requires an integration of the high-energy degrees of freedom, but the early proposals did just the opposite. In practice, integrating out low-energy modes leads to an infinite number of quantum corrections.

6. Y. Hatsugai and M. Kohmoto, "Exactly solvable model of correlated lattice electrons in any dimensions," J. Phys. Soc. Jpn 61, 2056 (1992).

7. P. W. Phillips et al., "Exact theory for superconductivity in a doped Mott insulator," Nat. Phys. 16, 1175 (2020).

8. Y. Gu et al., "Local criticality, diffusion and chaos in generalized Sachdev-Ye-Kitaev models," J. High Energy Phys. 2017, 125
(2017).

9. A. A. Patel et al., "Magnetotransport in a model of a disordered strange metal," Phys. Rev. X 8, 021049 (2018).

10. Y. Wang, "Solvable strong-coupling quantum-dot model with a non-Fermi-liquid pairing transition," Phys. Rev. Lett. 124, 017002 (2020); E. E. Aldape et al., "Solvable theory of a strange metal at the breakdown of a heavy Fermi liquid," arXiv:2012.00763.

11. N. G. Deshpande and X.-G. He, "Unparticle realization through continuous mass scale invariant theories," Phys. Rev. D 78, 055006 (2008). 